\title{
Anomalous Diffusion of Motile Colloids Dispersed in Liquid Crystals
}

\author{
Josep M. Pagès, Jordi Ignés-Mullol, and Francesc Sagués* \\ Departament de Ciència de Materials i Química Física, and Institut de Nanociència i Nanotecnologia $\left(I^{2} U B\right)$, \\ Universitat de Barcelona, Martí i Franquès 1, 08028 Barcelona, Catalonia, Spain
}

(Received 13 November 2018; published 15 May 2019)

\begin{abstract}
We study the superdiffusion of driven colloidal particles dispersed in a nematic liquid crystal. While motion is ballistic in the driving direction, our experiments show that transversal fluctuations become superdiffusive depending on the topological defect pattern around the inclusions. The phenomenon can be reproduced with different driving methods and propulsion speeds, while it is strongly dependent on particle size and temperature. We propose a mechanism based on the geometry of the liquid crystal backflow around the inclusions to justify the persistence of thermal fluctuations and to explain the observed temperature and particle size dependence of the superdiffusive behavior based on material and geometrical parameters.
\end{abstract}

DOI: 10.1103/PhysRevLett.122.198001

Introduction.-One of the classical features of dispersed colloids is their random motion, which arises from the intrinsic thermal fluctuations of the solvent where they reside [1]. Typically, this gives rise to normal diffusion, evidenced as a linear growth with time of the mean square particle displacements. This behavior is not universal, and it often breaks down, singularly when involving motion in structurally complex fluids. Anomalies are made apparent by either subdiffusion (sublinear growth) when diffusion is hindered by a crowded environment [2-4], or superdiffusion (superlinear growth) where fluctuations are made more persistent by the surrounding medium [5-7].

When colloids are dispersed in orientationally ordered liquids, such as a nematic liquid crystal (LC), Brownian motion is anisotropic [8,9]. Moreover, surface chemistry of the dispersed colloids determines the arrangement of the LC around the inclusions [10], which can lead also to anomalous diffusive behavior at short timescales that arises due to fluctuations in the LC orientational order [11].

A recent surge of interest in colloidal motion is related to understanding the physics of microswimming, either when referring to living species or to artificially engineered prototypes [12-14]. Studies have addressed the specific characteristic of complex dispersing media during the motion of active or driven inclusions [15-23]. In particular, recent simulations of active point particles moving in an anisotropic liquid $[24,25]$ show that the swimmers feature a superdiffusive behavior. In experiments, however, this result cannot be separated from the intrinsic distortions caused by the inclusions on the LC matrix, which alter the diffusion mode in Brownian particles [11] and are likely to play a role in active or driven scenarios.

In this Letter, we present experiments with the simpler case of driven, rather than active, colloidal particles moving in a nematic LC. We show that superdiffusive behavior arises depending on the boundary condition at the motile inclusion surface. We propose a mechanism for superdiffusion related to the backflow pattern (puller or pusher) around the particles [13] and show that the reported behavior does not depend on the propulsion mode when we compare sedimenting and phoretic colloids. We relate the change of the anomalous diffusion exponent with temperature and particle size to the amplitude and persistence of orientational fluctuations, which we estimate from material parameters and from the measured geometry of topological defects around the inclusions.

Experimental system.-We use spherical silica particles of diameter 5 and $10 \mu \mathrm{m}$ (Kisker Biotech). Homeotropic LC anchoring at the surface of colloidal particles, giving rise to single dipolar hyperbolic hedgehog defects, is promoted using specific coating protocols depending on the particle size. In particular, we employ $n$-octadecyltrichlorosilane (Gelest) for small particles, while dimethyl octadecyl [3-(trimethoxysilyl)propyl] ammonium (Sigma Aldrich) is used for the larger ones. Bare, nonfunctionalized particles, favoring planar anchoring, generate quadrupolar distortions in the form of dual surface point defects (boojums) [10]. The used LC is CCN-37 (Nematel) with negative dielectric anisotropy and a nematic to isotropic transition temperature $T_{N I} \simeq 55^{\circ} \mathrm{C}$. The LC in nematic phase is confined between two rubbed polyimide-coated (Nissan 825) bounding plates that provide planar anchoring conditions and a homogeneous far field. The typical cell thickness is $15 \mu \mathrm{m}$.

For the sedimentation experiment, the cell is tilted at an angle $\beta=8.5^{\circ}$ with respect to the horizontal, so that the particle velocity is parallel to the LC far-field director. The latter is a unit vector, $\hat{\boldsymbol{n}}$, that represents the local average orientation of LC molecules. In the alternative scenario, colloidal driving is induced through the well-known liquid 
crystal-enabled electrokinetic (LCEEK) mechanism [26-28]. Thin transparent electrodes coat the inner side of the LC cell plates in this case. Inside it, particles with dipolar distortion are propelled along the far-field director after application of an alternating electric field perpendicular to the plates. The overdamped motion of the colloids arises from uncompensated electro-osmotic flows permanently generated around them [28]. Although particle speed depends on the amplitude and frequency of the applied field, we choose to work with a fixed frequency which is specific for each particle size $(10 \mathrm{~Hz}$ for $5 \mu \mathrm{m}$; $50 \mathrm{~Hz}$ for $10 \mu \mathrm{m}$ particles) to achieve comparable speeds. Note that with the choice of a LC with negative dielectric anisotropy, out-of-plane fluctuations of the director are largely suppressed by the imposed electric field. The position of the centroid of moving particles is tracked automatically, using the open source software ImageJ, from micrographs acquired at rate of $1 \mathrm{fps}$ for sedimenting particles and $15 \mathrm{fps}$ for particles driven under LCEEK. Images are first binarized so that the particle contour can be clearly detected [29]. From the trajectories, we compute the mean displacement along the far-field director, $\left\langle\Delta r^{\|}(\tau)\right\rangle=1 / N_{\tau} \sum_{t=0}^{t=T-\tau}\left[r^{\|}(t+\tau)-r^{\|}(t)\right]$, and the mean square displacement (MSD) in the perpendicular direction, $\left\langle\left[\Delta r^{\perp}(\tau)\right]^{2}\right\rangle=1 / N_{\tau} \sum_{t=0}^{t=T-\tau}\left[r^{\perp}(t+\tau)-r^{\perp}(t)\right]^{2}$, where $N_{\tau}$ is the number of overlapping time intervals of width $\tau$ in the experimental time series.

Results. - The first set of results refers to the sedimentation of $5 \mu \mathrm{m}$ particles with dipolar LC distortion (see Fig. 1). Motion along the far-field director is essentially ballistic, with speeds of order $0.1 \mu \mathrm{m} \mathrm{s}^{-1}$. Diffusive scaling was resolved on time windows that span between the time needed for the particle to travel its own size and that needed to travel a distance equal to the cell thickness. For the conditions in Fig. 1, the analysis yields a superdiffusion exponent $\nu \approx 1.4$ for the transversal MSD.

Transversal superdiffusion was also found for sedimenting $10 \mu \mathrm{m}$ particles with dipolar LC distortion, although the evaluated exponents are notoriously larger (see Fig. 2), singularly at the smallest temperatures investigated. Although larger particles sediment at higher speed, this parameter has an undetectable influence on the transversal MSD, as shown below for particles under LCEEK driving [Fig. 2(b)]. Differences arising from particle size vanish at high temperatures (still well below the Nematic to Isotropic transition), and extracted $\nu$ values approach $\nu \approx 1.8$.

Benchmark experiments with the same sedimenting particles, performed by replacing the LC with a mixture of water and glycerol to match the viscosity of the LC at room temperature, were conducted to validate the experimental protocols, yielding the normal diffusive behavior $\nu \approx 1.0$ for transversal MSD (see the Supplemental Material [30]).

LCEEK-driven particles were investigated in a similar way, varying both particle size and temperature. Despite

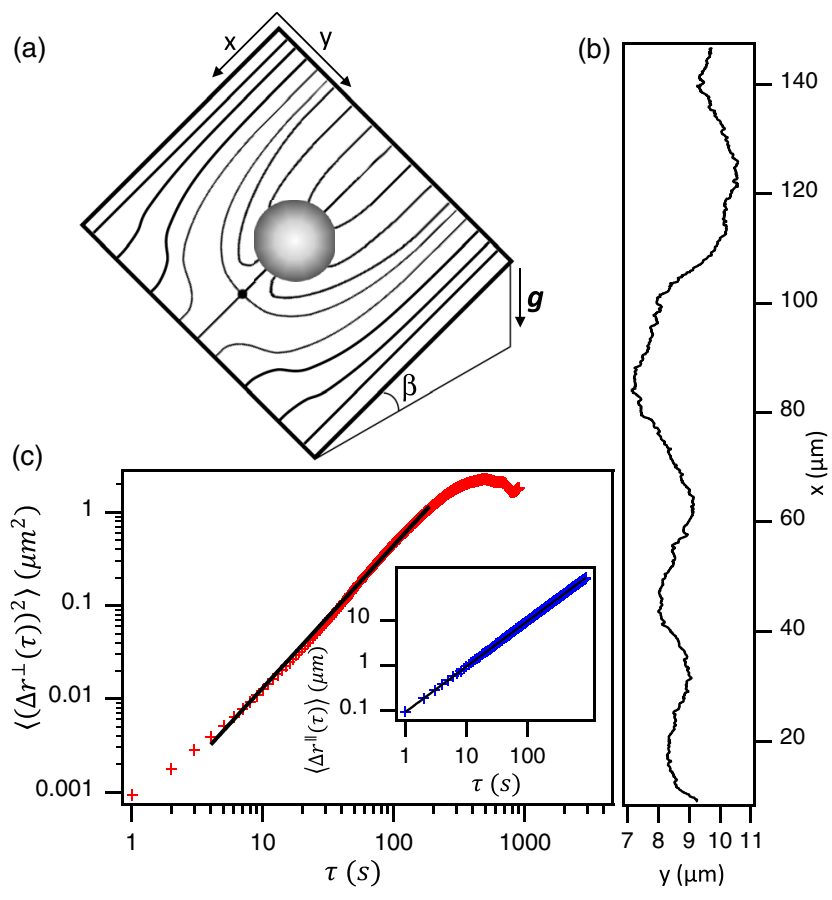

FIG. 1. Sedimentation of a $5 \mu \mathrm{m}$ particle with dipolar LC configuration. (a) Sketch of the experimental geometry and LC director surrounding the inclusion. (b) Particle trajectory in the $x / y$ plane at $T-T_{N I}=-27.5^{\circ} \mathrm{C}$. (c) MSD in the transversal direction vs time window. The solid line is a power law fit that yields an exponent $\nu=1.41 \pm 0.04$. In the inset, the mean parallel displacement is plotted vs time, showing a ballistic trend. The solid line has a slope 1 .

more than 2 orders of magnitude larger ballistic speeds, results for lateral displacement have shown analogous superdiffusion trends both with respect to particle size and temperature [see Fig. 2(a)]. In the case of the smaller particles, the applied electric field was tuned for each investigated temperature in such a way that the speed of colloidal motion was kept constant, although we verified that the ballistic speed does not influence the superdiffusive behavior in the used range of speeds [Fig. 2(b)]. Our studies are performed under strong confinement conditions, with the cell gap that is either 1.5 or 3 times larger than the particle diameters. Although the mismatch between LC anchoring on the particles and on the bounding plates should minimize surface drag, we have performed experiments with cell gaps of $60 \mu \mathrm{m}$, much larger than the particle diameters (see Fig. S2 of the Supplemental Material [30]). This results in a small enhancement of the superdiffusive behavior, showing that interaction with the substrate may dampen superdiffusion, but has no role in originating it.

To address the influence of the LC distortions around the particles, we repeated the series of sedimenting experiments but now replacing the functionalized colloids by bare particles that promote a planar anchoring. Notice that LCEEK drive is not a choice here, since the resulting 

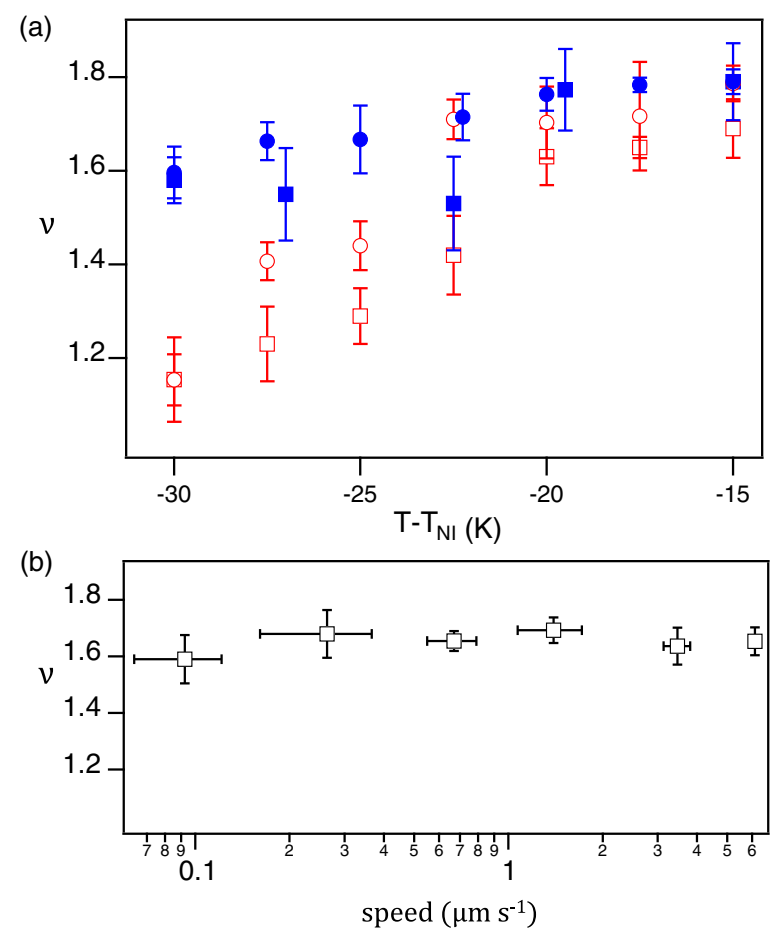

FIG. 2. (a) Temperature dependence of the superdiffusive exponent in the transversal MSD of nematic colloids featuring a dipolar configuration. Particles diameters are $10 \mu \mathrm{m}$ (blue, solid symbols) and $5 \mu \mathrm{m}$ (red, empty symbols), and driving mechanisms are sedimentation (circle) and electrokinetic (rectangle). (b) Exponents $(\nu)$ vs speed at $T-T_{N I}=-20{ }^{\circ} \mathrm{C}$ for $5 \mu \mathrm{m}$ electrokinetically-driven particles featuring a dipolar defect. Different speeds are achieved by tuning the electric field amplitude with a constant frequency of $10 \mathrm{~Hz}$. Error bars indicate the standard deviation over different experimental realizations.

quadrupolar defect structure prevents fore-aft symmetry breaking, which impedes this type of driven motion [28]. Results are summarized in Fig. 3. Compared to the case of particles with dipolar defects, exponents are now markedly smaller, and they barely differ from normal diffusion. This conclusion applies to the whole range of temperatures investigated.

Discussion.-We will next analyze the two main results of the reported experiments: the origin and temperature dependence of the superdiffusive behavior, and the relationship between the diffusive transport and the LC structure around a driven particle. This discussion is better framed by first comparing the timescales of the transport mechanisms involved in particle motion. Thermal fluctuations drive both erratic displacements, with an intrinsic timescale $\tau_{d} \sim\left(R^{2} / D\right)$, and random orientational fluctuations, evolving at a scale $\tau_{r} \sim\left(\gamma R^{2} / K\right)$. Here, $R$ is the radius of the spherical particles, $\gamma$ is the LC rotational viscosity, and $D$ and $K$ are, respectively, the average particle diffusion coefficient and the LC elastic constant, neglecting anisotropic effects. For micron-size particles and commonly employed LCs, $\tau_{d} \gg \tau_{r}$.
For driven colloids, random displacements and orientational fluctuations are advected by hydrodynamic flows at a characteristic timescale $\tau_{a} \sim\left(R / V_{0}\right)$, with $V_{0}$ denoting the colloid speed. Assuming again that $\tau_{d}$ is the largest timescale, the effect of hydrodynamics on the LC director can be assessed using the Ericksen number, $E_{r}=\tau_{r} / \tau_{a} \sim \gamma R V_{0} / K$, which compares viscous and elastic stresses. Large values indicate that the director field can be distorted by the backflow due to particle motion. Using characteristic values, representative of the whole range of our experimental conditions [31], we obtain $\tau_{r} \sim 0.1 \mathrm{~s}$ and $\tau_{a} \sim 10 \mathrm{~s}$. This results in $E_{r} \sim 10^{-2}$, indicating that director reorientations are much faster that the other processes at play. When comparing our experiments to those of anomalous diffusion in Brownian nematic colloids [11], we find that the latter are in the complementary regime, with director relaxation being the slowest process, suggesting that the physics of that system should be different from the one reported here.

Based on the discussion above, we conclude that particles in our system move along an essentially frozen nematic orientational field, $\hat{\boldsymbol{n}}$, within which a colloid may instantaneously misalign its direction of motion in a random manner. Such framework fits within the assumptions of both Lintuvuori et al. [33] and Daddi-Moussa-Ider et al. [34]. These authors argue that the misaligned director configuration is affected by a torque $f \propto V_{0} R^{2} \sin 2 \theta$ [33], where $\theta$ is the angle between the local director and the instantaneous particle velocity. The sign of $f$ depends on the flow pattern advected around the particles, namely, negative for pusherlike and positive for pullerlike flows [33]. Although such backflow patterns have not yet been established for the sedimentation case, they have been measured for LCEEK-driven colloids [28], showing that the flow is pullerlike in the presence of dipolar LC distortions and pusherlike in the quadrupolar case. As a result, we can conclude that the nematic torque would tend to keep the particles aligned with the far-field director for quadrupolar distortions while, contrarily, it enforces misalignment for dipolar distortions, thus promoting superdiffusive behavior in the latter case, consistently with our experimental observations.

The role on the superdiffusive behavior of the torque exerted on the misaligned director can be further demonstrated with the arguments raised by Despósito et al. [35,36]. Briefly, for transport in active complex media, the instantaneous particle motion perpendicular to $\hat{\boldsymbol{n}}$ is considered to obey a Langevin-like differential equation

$$
\gamma \frac{d r^{\perp}(t)}{d t}=f(t)+\xi(t)
$$

which expresses the balance between the friction drag, with $\gamma$ being the rotational viscosity, and two stochastic 

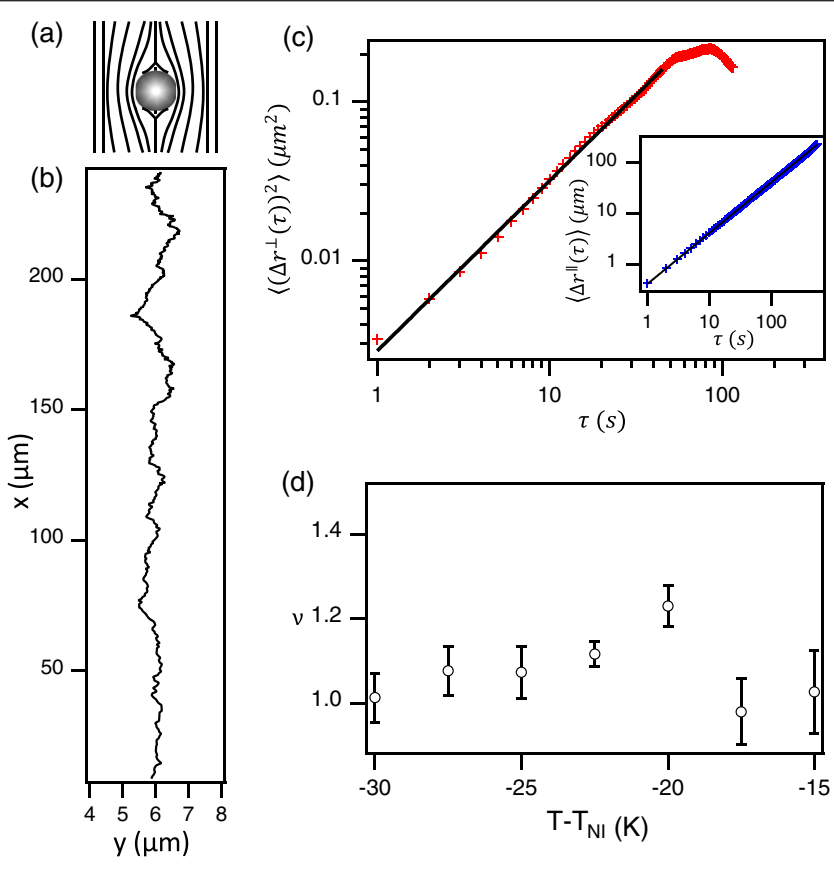

(a)
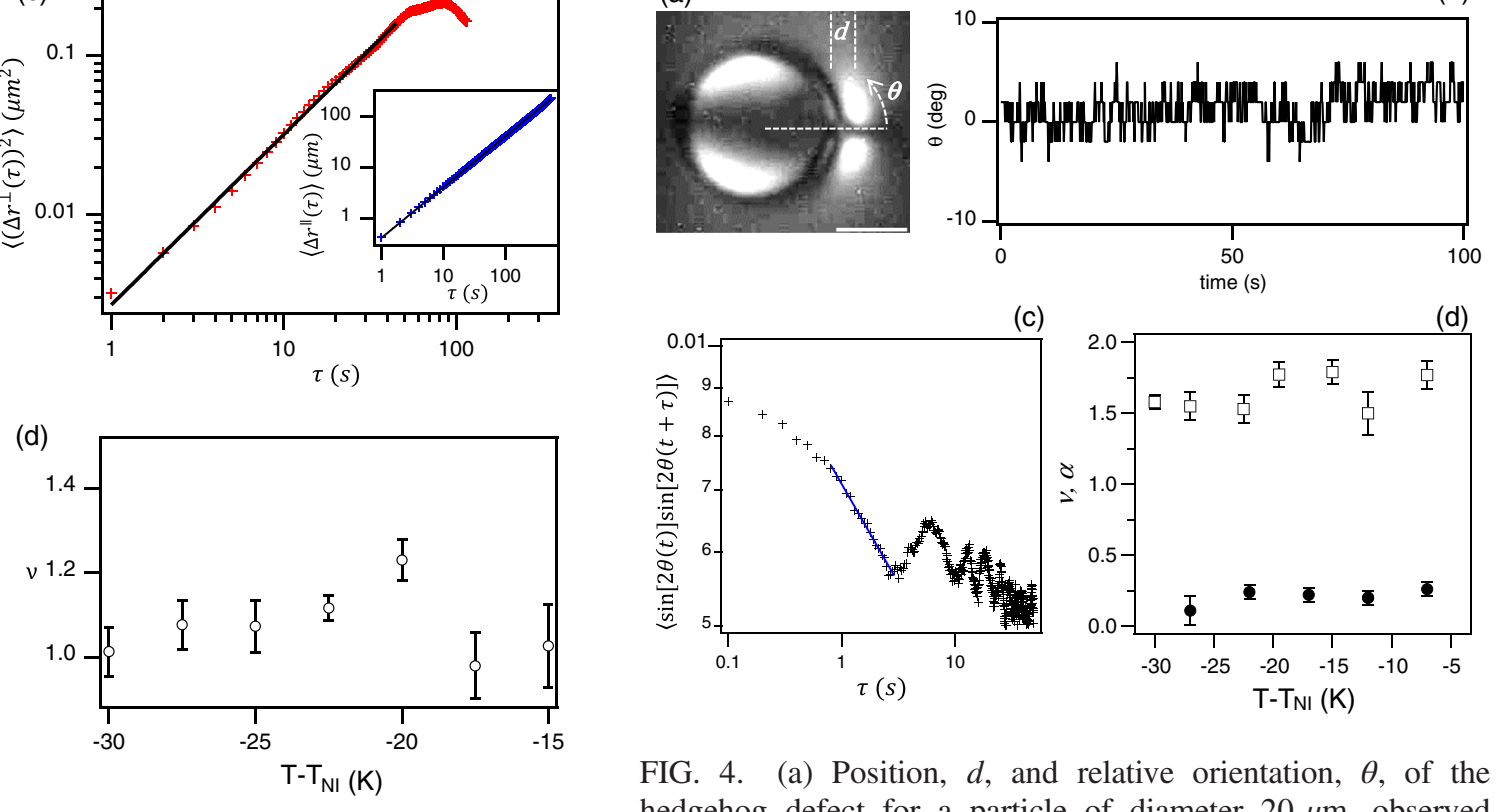

FIG. 3. Sedimentation of a $5 \mu \mathrm{m}$ particle with quadrupolar LC configuration. (a) Sketch of the LC director field around the inclusion. (b) Particle trajectory in the $x / y$ plane at $T-T_{N I}=-17.5^{\circ} \mathrm{C}$. (c) Mean square displacement in the transversal direction vs time window. The solid line is a power law fit that yields an exponent $\nu=1.0 \pm 0.1$. In the inset, the mean parallel displacement is plotted vs time, showing a ballistic trend. (d) Temperature dependence of the nearly diffusive exponent in the transversal MSD. Error bars indicate the standard deviation over different experimental realizations.

forces that operate on the particles. On the one hand, $\xi(t)$ represents the internal noise effects of thermal origin, for which a zero-mean white noise statistics is assumed. On the other hand, $f(t)$ introduces a source of stochasticity associated to the specific inherent dynamics of the nematic colloid. The latter is associated to the random kicks arising from the transient misalignment of the instantaneous particle velocity with respect to the far-field nematic director. According to our arguments above, $f(t)$ will be proportional to $\sin 2 \theta(t)$. The temporal fluctuations of this torque are, therefore, accessible in our experiments by monitoring the position of the distortion. In practice, the latter can only be reliably assessed for the $10 \mu \mathrm{m}$ particles, for which we have measured the defect distance to the particle surface, $d$ (a function of $T$ and $R$ ) and its orientation, $\theta(t)$ (Fig. 4). From these data, the statistical properties of $f(t)$ are obtained, in particular its time autocorrelation, $\langle f(t) f(t+\tau)\rangle=$ $\Lambda(|\tau|) \propto\langle\sin [2 \theta(t)] \sin [2 \theta(t+\tau)]\rangle$. Experimentally, we find that our data are compatible with a scaling of the type $\Lambda(\tau) \propto \Lambda_{0}\left(\tau / \tau_{0}\right)^{-\alpha} \cdot \Lambda_{0}$ measures the intensity of the intrinsic orientational fluctuations, and the positive exponent $\alpha$, according to our measurements, stays bounded and lower than unity, i.e., $0<\alpha<1$ [Figs. 4(c) and 4(d)]. Despósito et al. argue that, under these circumstances, the mean squared (c)

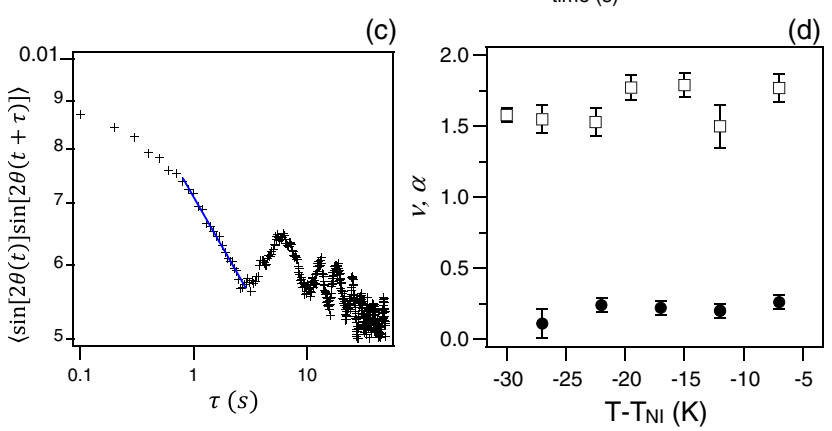

FIG. 4. (a) Position, $d$, and relative orientation, $\theta$, of the hedgehog defect for a particle of diameter $20 \mu \mathrm{m}$, observed between crossed polarizers, in a cell of thickness $25 \mu \mathrm{m}$. Scale, $10 \mu \mathrm{m}$. (b) Defect orientation with respect to the horizontal LC far field as a function of time for a particle of diameter $10 \mu \mathrm{m}$ under electrokinetic drive at $T-T_{N I}=-15^{\circ} \mathrm{C}$. (c) Autocorrelation of the driving torque, $f \propto \sin (2 \theta)$, for the data in (b). The solid line is a power law fit to $\langle f(t) f(t+\tau)\rangle \sim t^{-\alpha}$, with $\alpha=0.22$, for times larger than the time needed for the particle to travel its own size. (d) Exponent of the mean square displacement, $\nu$ (open rectangle), and force autocorrelation, $\alpha$ (filled circle), as a function of temperature, for $10 \mu \mathrm{m}$ particles under electrokinetic drive.

displacement associated to the generalized Langevin equation (1) reads [35,36]

$$
\begin{aligned}
\operatorname{MSD}(\tau) & =\left\langle\left[\Delta r^{\perp}(\tau)\right]^{2}\right\rangle \\
& =\frac{2 k_{B} T}{\gamma}\left(\left(\frac{\tau}{\tau_{0}}\right)+\epsilon \kappa_{1, \alpha}\left(\frac{\tau}{\tau_{0}}\right)^{2-\alpha}\right),
\end{aligned}
$$

where $\kappa_{1, \alpha}$ is a positive numerical factor, and $\epsilon$, denotes a dimensionless ratio between the two acting random forces. For $\alpha$ smaller than unity, the first purely diffusive contribution is subdominant in front of the superdiffusive term with exponent $2-\alpha$, so one should expect $\left\langle\left[\Delta r^{\perp}(\tau)\right]^{2}\right\rangle \simeq \tau^{2-\alpha}$ in the presence of superdiffusion in the transversal particle motion. When comparing $\nu$ (the transversal superdiffusion exponent, see Fig. 2) with $\alpha$, our results are consistent, within error bars, with the prediction $\alpha+\nu=2$, specially in the high temperature range [Fig. 4(d)].

The analysis above proves that the observed superdiffusive behavior is the result of the persistence in the misalignment of the dipolar director field surrounding the particles. However, it does not explain the observed increase in the superdiffusive exponent with particle size and with temperature. One could argue that the strength of 
the nematic torque should increase with the extension of the dipolar distortion, which is proportional to the inclusion size [37], and we have measured that it increases with temperature (see Fig. S3 of the Supplemental Material [30]). A complete analysis should also take into account the well-known temperature dependence of the nematic elasticity, which will condition the amplitude and dynamics of fluctuations. We have attempted a qualitative approach (see the Supplemental Material [30]) by assuming that the strength of this effect is related to the intensity of in-plane director fluctuations, being able this way to predict the observed temperature and size dependence (see Fig. S3 of the Supplemental Material [30]). Such assumption, however, is not rigorous, and more work is needed to fully understand the experimental results presented here.

Conclusions. - We have analyzed the anomalous diffusive transport of driven colloids dispersed in a nematic liquid crystal. Motion is ballistic along the driving direction while it can be superdiffusive in the transversal direction. The type of distortions of the elastic LC matrix around the moving colloids determines the transversal transport mode, the latter being diffusive for quadrupolar distortions and superdiffusive for dipolar distortions. These results do not depend significantly on driving speed or propulsion mode, while increasing temperature or particle size clearly enhances the anomalous diffusion. Based on existing models that analyze the dynamics of active particles in complex media, our experiments show a clear connection between superdiffusive motion and the persistence of thermal fluctuations endowed by dipolar liquid crystal distortions. These observations should prompt theoretical studies to substantiate our interpretations and conjectures, together with variants of the experiments here reported that may deepen our understanding of colloidal transport in anisotropic media both for active and driven scenarios.

J.M.P. acknowledges funding from the European Union's Horizon 2020 FET-Open "AbioMatter," Grant No. 665440. All authors acknowledge funding by MINECO (AEI/FEDER, EU), Projects No. FIS 201341144P and No. FIS 2016-78507-C2-1-P.

*f.sagues@ub.edu

[1] R. J. Hunter, Foundations of Colloid Science (Oxford University Press, Oxford, 2000).

[2] I. Y. Wong, M. L. Gardel, D. R. Reichman, E. R. Weeks, M. T. Valentine, A. R. Bausch, and D. A. Weitz, Phys. Rev. Lett. 92, 178101 (2004).

[3] J. Sprakel, J. van der Gucht, M. A. Cohen Stuart, and N. A. M. Besseling, Phys. Rev. E 77, 061502 (2008).

[4] M. M. Alam and R. Mezzenga, Langmuir 27, 6171 (2011).

[5] A. Ott, J. P. Bouchaud, D. Langevin, and W. Urbach, Phys. Rev. Lett. 65, 2201 (1990).

[6] X. L. Wu and A. Libchaber, Phys. Rev. Lett. 84, 3017 (2000).

[7] R. Angelico, A. Ceglie, U. Olsson, G. Palazzo, and L. Ambrosone, Phys. Rev. E 74, 031403 (2006).
[8] J. C. Loudet, P. Hanusse, and P. Poulin, Science 306, 1525 (2004).

[9] B. Senyuk, Q. Liu, S. He, R. D. Kamien, R. B. Kusner, T. C. Lubensky, and I. I. Smalyukh, Nature (London) 493, 200 (2013).

[10] I. Muševič, Liquid Crystal Colloids, Soft and Biological Matter (Springer, Cham, 2017).

[11] T. Turiv, I. Lazo, A. Brodin, B. Lev, V. Reiffenrath, V. Nazarenko, and O. Lavrentovich, Science 342, 1351 (2013).

[12] J. Elgeti, R. G. Winkler, and G. Gompper, Rep. Prog. Phys. 78, 056601 (2015).

[13] C. Bechinger, R. Di Leonardo, H. Löwen, C. Reichhardt, G. Volpe, and G. Volpe, Rev. Mod. Phys. 88, 045006 (2016).

[14] A. Zöttl and H. Stark, J. Phys. Condens. Matter 28, 253001 (2016).

[15] J. Schwarz-Linek, C. Valeriani, A. Cacciuto, M. E. Cates, D. Marenduzzo, A. N. Morozov, and W. C. K. Poon, Proc. Natl. Acad. Sci. U.S.A. 109, 4052 (2012).

[16] L. Zhu, E. Lauga, and L. Brandt, Phys. Fluids 24, 051902 (2012).

[17] T. Qiu, T. C. Lee, A. G. Mark, K. I. Morozov, R. Münster, O. Mierka, S. Turek, A. M. Leshansky, and P. Fischer, Nat. Commun. 5, 5119 (2014).

[18] S. Zhou, A. Sokolov, O. D. Lavrentovich, and I. S. Aranson, Proc. Natl. Acad. Sci. U.S.A. 111, 1265 (2014).

[19] M. S. Krieger, S. E. Spagnolie, and T. Powers, Soft Matter 11, 9115 (2015).

[20] A. Eremin, P. Hirankittiwong, N. Chattham, H. Nádasi, R. Stannarius, J. Limtrakul, O. Haba, K. Yonetake, and H. Takezoe, Proc. Natl. Acad. Sci. U.S.A. 112, 1716 (2015).

[21] J. R. Gomez-Solano, A. Blokhuis, and C. Bechinger, Phys. Rev. Lett. 116, 138301 (2016).

[22] A. E. Patteson, A. Gopinath, and P. E. Arratia, Curr. Opin. Colloid Interface Sci. 21, 86 (2016).

[23] C. Peng, T. Turiv, Y. Guo, Q. H. Wei, and O. D. Lavrentovich, Science 354, 882 (2016).

[24] J. Toner, H. Lowen, and H. H. Wensink, Phys. Rev. E 93, 062610 (2016).

[25] C. Ferreiro-Córdova, J. Toner, H. Löwen, and H. H. Wensink, Phys. Rev. E 97, 062606 (2018).

[26] O. D. Lavrentovich, I. Lazo, and O. P. Pishnyak, Nature (London) 467, 947 (2010).

[27] S. Hernandez-Navarro, P. Tierno, J. A. Farrera, J. IgnesMullol, and F. Sagues, Angew. Chem., Int. Ed. 53, 10696 (2014).

[28] I. Lazo, C. Peng, J. Xiang, S. V. Shiyanovskii, and O. D. Lavrentovich, Nat. Commun. 5, 5033 (2014).

[29] J. C. Crocker and D. G. Grier, J. Colloid Interface Sci. 179, 298 (1996).

[30] See Supplemental Material at http://link.aps.org/ supplemental/10.1103/PhysRevLett.122.198001 which contains a model for the temperature trend in the superdiffusion exponent, and figures with additional data and analysis.

[31] For CCN37, $\gamma$ is in the range $4-8 \times 10^{-2} \mathrm{Pas}, K$ is in the range $6-10 \mathrm{pN}$ [32], and our experiments feature speeds in the range $0.03-2 \mu \mathrm{m} \mathrm{s}^{-1}$. 
[32] P. Oswald, G. Poy, and A. Dequidt, Liq. Cryst. 44, 969 (2017).

[33] J. S. Lintuvuori, A. Würger, and K. Stratford, Phys. Rev. Lett. 119, 068001 (2017).

[34] A. Daddi-Moussa-Ider and A. M. Menzel, Phys. Rev. Fluids 3, 094102 (2018).
[35] L. Bruno, V. Levi, M. Brunstein, and M. A. Desposito, Phys. Rev. E 80, 011912 (2009).

[36] M. A. Despósito, C. Pallavicini, V. Levi, and L. Bruno, Physica (Amsterdam) 390A, 1026 (2011).

[37] P. Poulin, H. Stark, T. Lubensky, and D. A. Weitz, Science 275, 1770 (1997). 


\title{
Anomalous diffusion of motile colloids dispersed in liquid crystals
}

\author{
Josep M. Pagès, Jordi Ignés-Mullol, and Francesc Sagués
}

March 22, 2019

\section{Model for the temperature trend in the superdiffusion exponent}

We finally interpret, albeit in a qualitative way, the observed trends of the anomalous exponents when changing both colloid radius and temperature. Superdiffusion is the result of a persistence of the colloid misalignment that transiently prevails over thermal randomization. We propose a way to estimate the strength of this effect by looking at the intensity of in-plane director fluctuations $\left\langle(\delta \hat{\boldsymbol{n}})^{2}\right\rangle$ relative to the Boltzmann factor $\left(k_{B} T\right)$. By inverse Fourier transforming the director correlation function under the one-constant approximation, this ratio is expressed as [1]

$$
\frac{\left\langle(\delta \hat{\boldsymbol{n}})^{2}\right\rangle}{\left(k_{B} T\right)} \propto \frac{1}{K h} \int \frac{\boldsymbol{d q}}{q^{2}} \simeq \frac{1}{K h} \int_{\delta^{-1}}^{l^{-1}} \frac{d q}{q}=\frac{1}{K h} \ln \frac{\delta}{l},
$$

where $h$ stands for the cell thickness, $\delta(R)$ is taken as the typical length over which orientational fluctuations spread around a colloid of radius $R$, and $l$ is a cut-off setting the minimum length-scale that justifies a continuum description of the nematic elasticity. This calculation yields $\nu \sim \ln (\delta) / K$, which depends implicity on temperature both through $\delta$ and $K$. We assume that $\delta$ is proportional to the extension of the hedgehog defect, $d$, which we observe to increase with temperature (Fig. 3). Using the temperature dependence of $K$ reported in the literature [2], we find that the trend experimentally observed for $\nu$ is consistent with the above theoretical prediction (see Fig. 3). Within this same framework, the observation that $\nu$ is consistently larger for larger particles (see main text) can be justified by the well-known fact that the range of LC distortions is proportional to the inclusion size [3].

\section{References}

[1] P. G. de Gennes and J. Prost, The physics of liquid crystals, Oxford science publications (Clarendon Press ; Oxford University Press, Oxford New York, 1993), 2nd ed.

[2] P. Oswald, G. Poy, and A. Dequidt, Liquid Crystals 44, 969 (2016).

[3] P. Poulin, H. Stark, T. Lubensky, and D. A. Weitz, Science 275, 1770 (1997). 


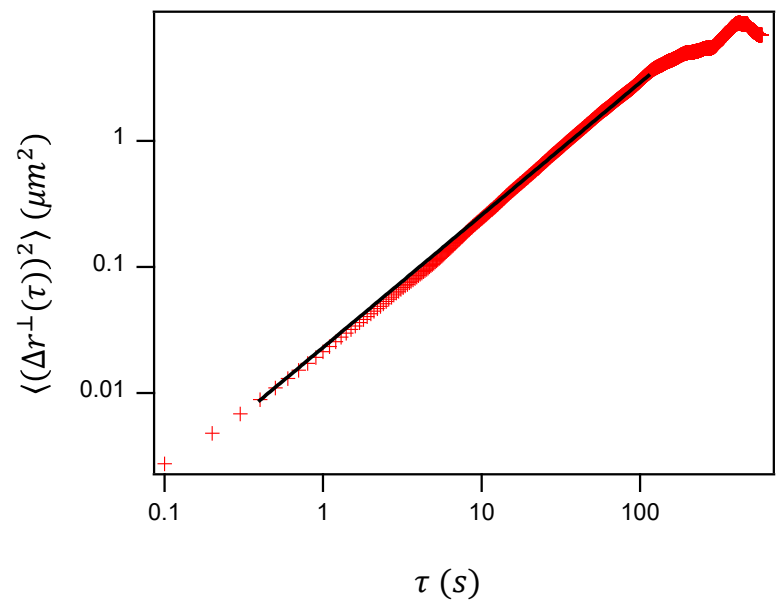

Figure S1: Mean square displacement of the transversal fluctuations vs. time window for an spherical particle of radius $4.7 \mu \mathrm{m}$ sedimenting though a water/glycerol mixture $(20 \% \mathrm{w} / \mathrm{w})$ in a glass cell of spacing $24 \mu \mathrm{m}$, and tilted at an angle of $8.5^{\circ}$. The solid line is power law fit to the data with exponent $1.05 \pm 0.02$.

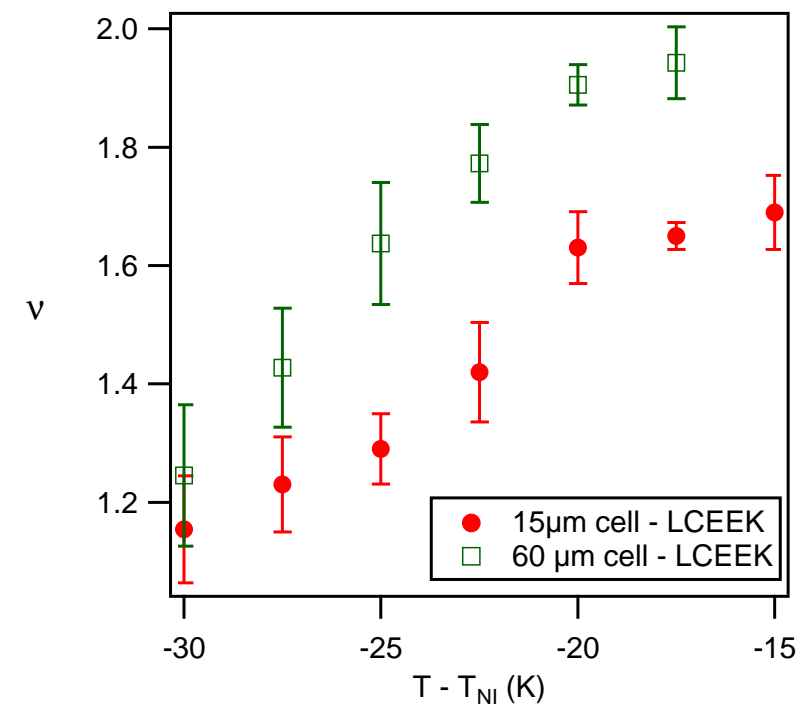

Figure S2: Exponent of the MSD for transversal fluctuations for $5 \mu \mathrm{m}$ particles with dipolar LC configuration, driven by LCEEK. Comparison of two series of experiments performed with LC cells with different gap thickness. 
(a)

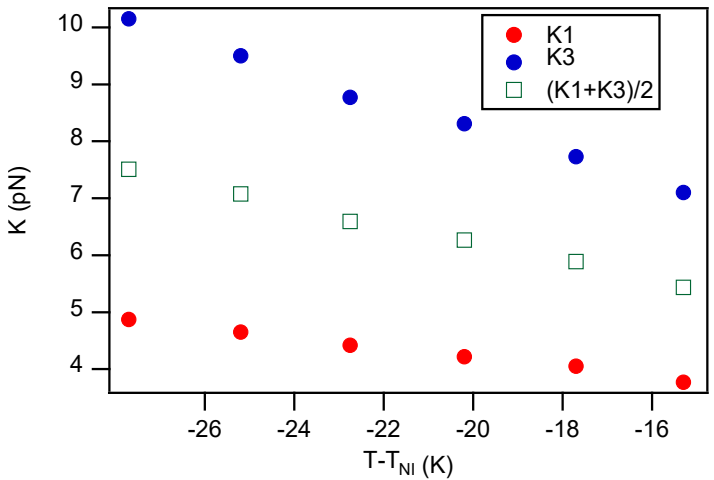

(b)

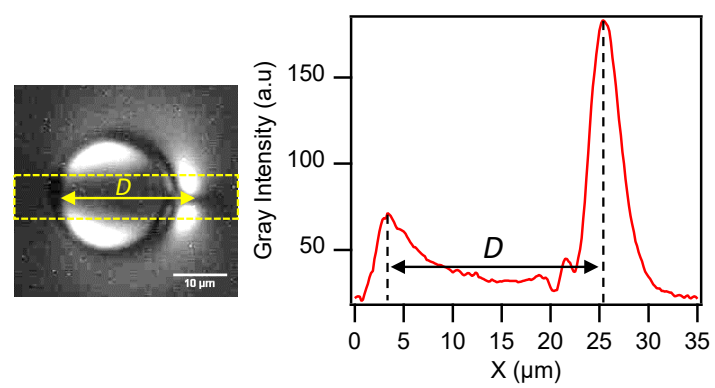

(c)

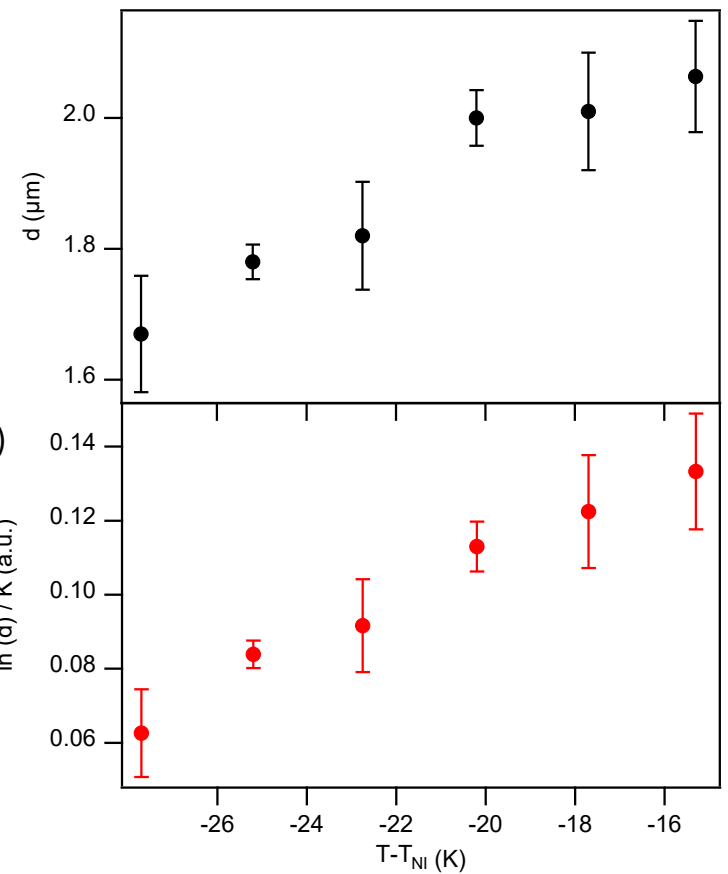

Figure S3: (a) Temperature dependence of the splay $\left(K_{1}\right)$ and bend $\left(K_{3}\right)$ elastic constants of CCN37 [2]. For the single constant approximation we use the arithmetic mean of K1 and K3. (b) The distance from the defect to the particle surface is determined from the grayscale profile in a thick band along the diameter of the particle. Under these imaging conditions, the position of the defect core results in a maximum average intensity, while the locations across the particle diameter exhibits another local maximum intensity. Here, a particle with diameter $2 R=20 \mu \mathrm{m}$, in a cell of thickness $25 \mu \mathrm{m}$ is analyzed. (c) Change with temperature of the distance between the particle surface, $d=D-2 R$, and the defect of the dipolar distortion. (d) Computed temperature evolution of the scaling prediction for the anomalous diffusion exponent, $\nu \sim \ln (d) / K$. 